\title{
Wolf FEUERHAHN (dir.), La Politique des chaires au Collège de France
}

\section{Philippe Moreau}

\section{(2) OpenEdition \\ 1 Journals}

Édition électronique

URL : https://journals.openedition.org/ress/4104

DOI : $10.4000 /$ ress. 4104

ISSN : 1663-4446

Éditeur

Librairie Droz

\section{Édition imprimée}

Date de publication : 14 décembre 2018

Pagination : 275-279

ISSN : 0048-8046

\section{Référence électronique}

Philippe Moreau, «Wolf FEUERHAHN (dir.), La Politique des chaires au Collège de France », Revue

européenne des sciences sociales [En ligne], 56-2 | 2018, mis en ligne le 14 décembre 2018, consulté le 08 janvier 2022. URL : http://journals.openedition.org/ress/4104 ; DOI : https://doi.org/10.4000/ress. 4104

Ce document a été généré automatiquement le 8 janvier 2022.

(c) Librairie Droz 


\title{
Wolf FEUERHAHN (dir.), La Politique des chaires au Collège de France
}

\author{
Philippe Moreau
}

\section{RÉFÉRENCE}

Wolf FEUERHAHN (dir.), 2017, La Politique des chaires au Collège de France, Paris, Collège de France / Les Belles Lettres, « Docet omnia », 560 p.

1 «Répondant à l'actualité migratoire, le Collège de France a décidé de créer une chaire "Migrations et Sociétés", qu'il confie à François Héran ". C'est ainsi que le quotidien français Libération a rendu compte en avril 2018 d'une élection dans cette prestigieuse institution. Sans trop forcer le trait, on pourrait dire que les trois caractéristiques généralement associées à cet établissement sont rappelées avec une belle concision : le souci de suivre au plus près l'évolution des phénomènes et des recherches scientifiques qui les prennent pour objet, en accordant à ces dernières le statut de discipline et une reconnaissance institutionnelle, l'autonomie des membres de l'institution dans la détermination des enseignements qui y seront dispensés, le primat (théorique), chronologique et procédural, du choix d'un enseignement sur celui de la personne qui l'assurera. Telle est d'ailleurs l'image que l'institution a répétitivement donnée d'ellemême, ce qu'une contributrice nomme, par une métaphore ethnologique légèrement caustique, "le discours indigène ». C'est donc à deux aspects statutairement liés du fonctionnement de cet établissement, la définition des disciplines et le recrutement des enseignants, qu'est consacré ce gros volume dirigé par Wolf Feuerhahn issu d'un colloque tenu au Collège en 2014 dans le cadre d'un projet de recherche « Passage des disciplines : histoire globale du Collège de France XIX ${ }^{\mathrm{e}}-\mathrm{XX}^{\mathrm{e}}$ siècles », initié en 2012 par Antoine Compagnon, qui y professe : c'est dire que l'ouvrage témoigne de la recherche réflexive et introspective que mène sur elle-même une institution, ce qui, en théorie, n'est pas sans risque, le plus important n'étant sans doute pas celui du "geste iconoclaste " (pour reprendre l'expression de Feuerhahn, p. 16). Il semble, au vu du projet d'ensemble et de la nature du présent volume, que le Collège de France fournisse 
l'objet d'une case study destinée à une «histoire intellectuelle, politique et institutionnelle de la France » (Feuerhahn), étude que l'on pourrait considérer comme marginale si l'on raisonne en termes de poids démographique de l'institution, comparée aux Universités et à un moindre degré aux Écoles, mais qui se révèle, à la lecture, un observatoire particulièrement fructueux, vu la centralité intellectuelle que confère au Collège son prestige.

2 L'entreprise relève donc de l'histoire et de la sociologie des institutions, non seulement de l'histoire interne d'un corps académique, mais aussi de ses relations avec d'autres, porteurs de missions voisines, et elle combine analyse des règles, généralement dictées par le pouvoir d'État - et du fonctionnement concret, dans le cadre de celles-ci, d'un groupe social pris dans son ensemble -, et série de "fiches" individuelles de professeurs ou de candidats malheureux (souvent révélatrices des débats et tensions dans l'institution), dans une démarche prosopographique. La recherche appartient également à l'histoire des sciences et à l'épistémologie, puisque son but ultime est de comprendre comment naissent, se transforment, meurent parfois, les savoirs institués en disciplines. Ces deux perspectives intellectuelles sont à l'évidence indissolublement liées, puisque le jeu interne des définitions et redéfinitions disciplinaires ne s'est naturellement pas exercé en vase clos, mais en interaction avec les mouvements, également complexes, des autres grandes institutions académiques, l'Université essentiellement, mais aussi le Jardin du Roi (devenu Museum) et l'observatoire, sous l'Ancien Régime, auxquels sont venus s'ajouter les Écoles : École pratique des hautes études au XIX ${ }^{\mathrm{e}}$ siècle et École des hautes études en sciences sociales au XX' . De même, le volume met en lumière le rôle des divers contextes politiques (on songe bien sûr à la fameuse révocation de Michelet en 1852) et idéologiques, dont le plus étonnant pour le profane est sans doute le phénomène de création de chaires " coloniales ", publiques ou privées, dans la France de la III République (voir l'article de Florence Deprest).

Outre une préface de Compagnon et une introduction de Feuerhahn, laquelle tout à la fois présente la problématique et la méthodologie de l'ouvrage et remédie en partie à l'absence de synthèse conclusive, le livre est constitué de seize contributions (toutes accessibles en libre accès à l'adresse suivante: https://books.openedition.org/ lesbelleslettres/120), individuelles ou collectives, dues à vingt auteurs (dont le profil scientifique est présenté pages 535-541), essentiellement des historiens des sciences, avec quelques sociologues. Les articles sont regroupés en quatre parties : "Anciens et nouveaux régimes des chaires ", prenant en compte les effets de la coupure advenue à la fin du XVIII ${ }^{e}$ siècle, "Idéaux et pratiques de la transformation des intitulés ", centrée sur le recrutement des personnes et ses déterminations, l'hérédité biologique ou intellectuelle, l'appartenance à l'institution, le contexte idéologique, le poids croissant des laboratoires, «Le poids des "chaires fondamentales" " (au sens de: existantes depuis la fondation), présentant l'évolution de quatre disciplines, grec, latin, archéologie, mathématiques, et enfin "Intitulés et disciplines : jeux de masques », au cœur de la problématique choisie, s'attachant à la complexité des stratégies articulant disciplines et intitulés. La conservation dans le volume de l'architecture d'un colloque rassemblant de nombreux participants génère ses effets habituels, positifs (le croisement d'une pluralité de points de vue et d'approches) et négatifs (ainsi, quelques redites dans le rappel des points d'histoire, comme le contenu du décret réglementaire de 1873, ou l'analyse de la fameuse formule lancée par Renan en 1868 sur la « science en voie de se faire »). 
4 Le corpus documentaire sur lequel se fondent les contributions comprend essentiellement des documents officiels, strictement formalisés, issus des administrations de l'État ou de l'institution elle-même (voir l'Avertissement, p. 13, et les indications référencées systématiquement données par chaque auteur), sources archivistiques conservées au Collège : registres de délibérations et procès-verbaux des assemblées des professeurs, dossiers des professeurs et des chaires (ces derniers permettant l'élaboration des fiches prosopographiques). Le programme de recherche «Passage des disciplines » se déroule parallèlement à une entreprise de numérisation de ce fonds local. La nature et l'origine de ces sources, quelle que soit leur très grande valeur, implique, par le conformisme de l'expression et de la pensée propre aux documents officiels, le risque de ne saisir qu'une histoire idéologique, utile mais assez convenue: les intervenants dans les assemblées de professeurs invoquent ainsi régulièrement, comme un credo, la définition et les principes reconnus. En outre, l'assemblée des professeurs a décidé en 1873 de n'enregistrer dans les procès-verbaux que des résumés des débats, décision «bien dommageable pour l'historien " note Hélène Gispert (par ailleurs légitimement sensible aux biais des sources). Le meilleur remède est le recours, pratiqué par plusieurs contributeurs, à une documentation privée, essentiellement des mémoires, autobiographies et correspondances (par exemple dans les contributions de Françoise Waquet, Pascale Rabault-Feuerhahn, Olivier Orain et Marie-Claire Robic), qui fournissent un contrepoint, en quelque sorte, en les commentant de manière parfois crue et acerbe, aux comptes rendus officiels des débats institutionnels et qui permettent des analyses croisées. La palme dans ce domaine revient à l'admirable correspondance des deux historiens fondateurs des Annales, Marc Bloch et Lucien Febvre, publiée entre 1994 et 2003, dont la liberté d'expression et la franchise souvent rude sur les personnes créent un fort effet de contraste, allant jusqu'à la remise en cause d'un des traits fondateurs de l'institution : l'absence de division en facultés disciplinaires (sciences, lettres, droit, etc.), ayant pour effet, apparemment paradoxal, de confier à une forte majorité de non-spécialistes le recrutement d'un spécialiste, et donnant aux rapporteurs des candidatures un pouvoir déterminant. Une remarque ironique de Febvre dans une lettre (d'ailleurs confirmée par une intervention publique dans un débat interne: voir Waquet, p.424) dément l'optimisme de Renan sur ce point (voir Renisio, p. 229, n. 14).

5 La période étudiée s'étend en principe de la fondation en 1530 à nos jours, et le Collège des lecteurs royaux de l'Ancien Régime, encore fortement marqué par l'hérédité des chaires conforme au modèle social dominant et par une certaine permanence des chaires (la transformation des intitulés ne devient la règle qu'à la fin du XVIII ${ }^{\mathrm{e}}$ siècle) est étudié par Rafael Mandressi et Jeanne Peiffer, mais en fait le centre de gravité se trouve aux $\mathrm{XIX}^{\mathrm{e}}$ et $\mathrm{XX}^{\mathrm{e}}$ siècle. La scansion historique est fournie par les réformes qu'imposèrent les pouvoirs publics: les lettres patentes de 1772 , résultant de la pression exercée par l'université de Paris et de la volonté réformatrice d'un ministre éclairé, le comte de Saint-Florentin; puis diverses modifications réglementaires en $1806,1829,1852,1857$, avant le grand décret de 1873 instaurant la chronologie des deux votes (sur le maintien, la transformation de la chaire ou la création d'une autre ; puis l'élection d'un professeur), qui sera maintenue par le décret de 1901.

6 La « chaire » professorale est bien l'unité fonctionnelle de base du système, même si on voit apparaître, en second plan, d'autres enseignants que les professeurs: les suppléants, aux XVIII ${ }^{\mathrm{e}}$ et $\mathrm{XIX}^{\mathrm{e}}$ siècle, les assistants, au XX ${ }^{\mathrm{e}}$, et surtout le personnel d'une 
nouvelle structure incluse, celle des laboratoires, qui d'une part joue un rôle de plus en plus important dans les sciences dures - en tant qu'élément incitant à la permanence des chaires auxquelles ils sont rattachés, par un légitime souci de rentabilisation des investissements financiers et humains -, et d'autre part introduit dans le Collège un modèle identique à celui qui fonctionne dans les Universités et les Écoles. D'où l'importance centrale des intitulés de ces chaires (résultant d'une interaction entre les vœux des candidats et la décision de l'assemblée des professeurs), choisis par les instigateurs de l'enquête comme angle de visée et voie d'approche, qu'il importe donc d'étudier jusque dans leur formulation littérale. Plusieurs contributions mettent en lumière des récurrences terminologiques par périodes, révélatrices de la mise en place de courants intellectuels dominants: "civilisation", "comparé», "structure », et présentent des remarques partielles, selon la plus ou moins grande sensibilité des auteurs, historiens et sociologues, comme on l'a vu, à l'aspect linguistique de la formulation même des intitulés (les contributrices les plus attentives à ce point sont Vivi Perraky et Élise Lehoux). S'il est vrai que sont ainsi signalées leur longueur, croissante au fil du temps, la référence à des périodes, à des zones géographiques, à des méthodes scientifiques, etc., la complexification des énoncés, du simple lexème au syntagme, complété d'un nombre plus ou moins important de circonstants (par exemple de temps et de lieu), il n'en demeure pas moins que l'on ressent le manque d'un bref développement (qui aurait pu être confié à un linguiste stylisticien) prenant en compte globalement et transversalement ces intitulés en tant qu'énoncés, et systématisant et périodisant les relevés occasionnels de traits spécifiques.

7 Le volume est remarquablement fabriqué (lire cependant p. 384 «de pair»; p. 376 et 521 «Legouvé»), et muni des instruments auxiliaires habituels : une bibliographie générale, un index des noms (où domine largement celui de Renan, ce qui confirme son statut de figure de référence de l'institution), une table des vingt-cinq figures. Sa lecture n'est pas toujours aisée, en raison de sa nature même de rassemblement de contributions et de la densité de certains textes. On conseillera de l'aborder, après l'introduction, par l'article de Yann Renisio, «Les crédits des chaires. Inertie disciplinaire et ascension des sciences au Collège de France (1800-2000) », qui présente avec clarté l'essentiel de la problématique (on signalera son diagramme de l'évolution des chaires sur deux siècles, p.232-233, de lecture difficile, mais qui visualise efficacement la question centrale). Enfin, on se rappellera la nature du projet : fournir des matériaux à une enquête plus vaste, ce qui implique que le temps des conclusions n'est pas encore venu. En ce sens, on doit considérer que ce volume remplit avec succès la mission que ses promoteurs lui avaient assignée.

\section{AUTEURS}

\section{PHILIPPE MOREAU}

Université Paris-Est Créteil, ANHIMA 